\title{
Espacio público: en busca de nuevos significados y estrategias de sustentabilidad social. Un estudio comparado de dos barrios $^{*}$
}

\author{
Rocío Valderrama Fabres**
}

\begin{abstract}
El presente artículo da cuenta de los principales resultados y reflexiones de un proceso de intervención e investigación desarrollado en torno a la percepción que los residentes de dos barrios tienen del espacio público, las imágenes asociadas a este espacio y si se constituye como una dimensión importante en la configuración de su ciudad; todo ello, considerando el contexto de construcción de estos espacios. Este estudio se realiza asumiendo que lo público se define a partir de los usos del espacio y no a partir de su estatuto jurídico, relevando la importancia de los significados que le asignan los sujetos. Los principales resultados de este proceso, se relacionan con la configuración local de las ciudades en donde las relaciones que se establecen con el territorio y con lo público se ven permeadas por las experiencias del habitar de cada sujeto. Al mismo tiempo, dependiendo de la relación que se establece con el territorio, es posible identificar estrategias en torno a la sustentabilidad de estos espacios, no solo en términos estructurales sino que también, en términos sociales y simbólicos.
\end{abstract}

Palabras clave: Ciudad, espacio público, sustentabilidad social.

\section{Espaço público: na procura de novos significados e estratégias de sustentabilidade social. \\ Um estudo comparativo de dois bairros}

\section{RESUMO}

O presente artigo da conta dos principais resultados e reflexões de um processo de intervenção e investigação desenvolvido em torno à percepção que os moradores de dois bairros têm do espaço público, as imagens associadas a este espaço e se estas se constituem como uma dimensão importante na

Artículo recibido el 4/08/2015. Artículo aprobado el 2/10/2015.

** Chilena. Trabajadora Social, Fundación Mi Parque. E-mail: rvalderrama@ miparque.cl 
Espacio público: en busca de nuevos significados y estrategias de sustentabilidad social. Un estudio comparado de dos barrios / Valderrama

configuração de sua cidade; tudo isto, considerando o contexto de construção destes espaços. Este estudo foi realizado assumindo que o público é definido a partir dos usos dos espaços e não do seu estatuto jurídico, relevando a importância dos significados atribuídos pelos indivíduos. Os principais resultados deste processo relacionam-se com a configuração local das cidades onde as relações que se estabelecem com o território e com o público são permeadas pelas experiências do habitar de cada sujeito. Ao mesmo tempo, dependendo da relação estabelecida com o território, é possível identificar estratégias em torno à sustentabilidade desses espaços, não só em termos estruturais, mas também em termos sociais e simbólicos.

Palavras-chave: Cidade, espaço público, sustentabilidade social.

\title{
Public Spaces: in search of new meanings and strategies in social sustainability.
} A comparative study of two neighborhoods

\begin{abstract}
The present article accounts for the main results and reflections taken from an intervention and research process developed from the perception that residents from two neighborhoods have of public space, images associated with this space and whether this perception constitutes an important dimension in the configuration of their city; all of this taking into account the context of the construction of these spaces. This study takes place assuming that public is defined by the use of the public space and not by its legal status, revealing the importance of the meanings given to pubic space by people. The main results of this process are related to the local configuration of cities, where relationships established with the territory and with the public are permeated by the living experiences of each person. At the same time, depending on the relationship established with the territory, it is possible to identify strategies around the sustainability of these spaces, not just in structural terms but also in social and symbolic terms.
\end{abstract}

Key words: City, public spaces, social sustainability

\section{Introducción}

El presente artículo da cuenta de los resultados de un proceso de investigación e intervención desarrollado el año $2013^{1}$ en Fundación Mi Parque ${ }^{2}$, organización que busca crear comunidad a tra-

Proyectos ejecutados por la autora del presente artículo.

2 Fundación que surge el año 2007 por la preocupación de un grupo de profesionales respecto al espacio cotidiano donde las personas habitan, 
vés de la recuperación participativa de áreas verdes en barrios donde estos espacios se distribuyen inequitativamente en relación al Área Metropolitana de Santiago. El objetivo planteado en la investigación fue "conocer la percepción de los residentes de la Villa San Luis de Renca y la Villa El Almendral de Puente Alto $^{3}$ respecto al espacio público como elemento estructurador del barrio". Este análisis se realiza a partir del proyecto ejecutado por Fundación Mi Parque en ambas villas, el cual consistió en la recuperación y reconstrucción del espacio público ubicado en cada sector a través del diseño participativo; este consiste en el desarrollo de talleres con el objetivo de definir (junto a las comunidades) el diseño de la plaza en base a la historia del barrio y usos de este espacio, y definir estrategias de cuidado y mantención. Paralelamente, como objetivo de intervención se busca "conocer el discurso de los vecinos de la Villa San Luis de Renca respecto a la reconstrucción del espacio público que habitan”. Ambos procesos surgen por la necesidad de contribuir en la incorporación de dimensiones subjetivas en la intervención territorial que realiza Fundación Mi Parque.

La relevancia de este proceso radica en cómo entendemos el espacio público y los significados que adquieren estos espacios según el sujeto que los habita. En este caso, lo público se observa como factor de centralidad, como elemento estructurador de la imagen de ciudad y barrio, en donde la calidad depende de la intensidad de usos que promueve, es decir lo público se define a partir de los usos del espacio y no de su estatuto jurídico (Borja y Muxi, 2003). Este estudio releva la dimensión social de los espacios públicos a través de los distintos significados que adquiere este espacio y su dimensión física, como un elemento estructurador, el que se

planteando como objetivo mejorar las áreas verdes del "Chile urbano" junto a las comunidades involucradas. Actualmente, existen distintas categorías de proyectos, desarrollando intervenciones en plazas, recintos educacionales y planes maestros.

3 Ambas comunas se ubican en la periferia del Área Metropolitana de Santiago, concentrando gran cantidad de viviendas sociales. 
entiende a través de la configuración de las categorías sendas, barrios, nodos (núcleos) y bordes ${ }^{4}$.

El diseño metodológico de la investigación fue de carácter cualitativo desde un enfoque de participación comunitaria. Este enfoque permitió reconocer la experiencia de los sujetos como parte de la construcción del fenómeno abordado. Las técnicas de recolección de información corresponden a entrevistas en profundidad, las cuales se complementan con mapas o cartografía social, para obtener una imagen del territorio que habita; estos mapas fueron realizados por las personas entrevistadas que residen en la Villa San Luis (Renca) y la Villa el Almendral (Puente Alto). De esta forma, se busca comprender sus percepciones respecto a su experiencia y vivencia del barrio y el espacio público. Al mismo tiempo, a través de los mapas se busca caracterizar el barrio, identificando si el espacio público puede considerarse como elemento estructurador del territorio.

Los principales hallazgos de este proceso se relacionan con la configuración local que hacen los sujetos respecto al espacio vivido, donde las experiencias de cada uno en torno al habitar van dotando de significado el territorio y lo público. De esta forma, el espacio reconstruido junto a Fundación Mi Parque, alcanza distintas connotaciones: en la Villa San Luis, adquiere el carácter de borde, actúa como límite entre sectores que se consideran seguros o inseguros, dónde se puede transitar o no. Mientras que en la villa El Almendral el espacio reconstruido se percibe como un nodo, es decir, adquiere un carácter central en la imagen del territorio, desde donde se configuran distintos significados respecto al barrio y la relación con un otro. Esta imagen permite identificar distintas estrategias de susten-

Las sendas son los conductos que siguen las personas regular u ocasionalmente y que son capaces de organizar y conectar los otros elementos, los barrios corresponden a aquellas zonas que las personas pueden ingresar a través de su pensamiento y que tiene carácter compartido, los bordes son aquellos elementos fronterizos que el sujeto no usa, son líneas que pueden separar o unir dos sectores, los nodos son los elementos estratégicos de la ciudad donde los sujetos pueden ingresar, pueden constituir el centro de un barrio o convergencias de calles (Lynch, 1998). 
tabilidad social, las cuales estarían permeadas por las percepciones que se configuran dentro de este espacio local.

\section{Ciudad y Espacio Público}

Durante las últimas décadas la preocupación por la vida urbana, la ciudad y los espacios ha ido en aumento. Esta discusión tendría su origen con las primeras manifestaciones y efectos que tuvo la aplicación del sistema neoliberal en todo el mundo. Reflejo de ello es Chile, país pionero en adoptar este tipo de medidas, donde a partir de 1979 las ciudades se observan desde una lógica de mercado, competitividad y rentabilización del suelo (De Mattos, 2004; Rodríguez A. y Rodríguez P., 2012). Las transformaciones que arrastra este proceso han sido diversas, sin embargo, es preciso detenerse en las consecuencias, por una parte, de una "morfología social donde persiste la polarización social y la segregación; y, por otra, de una morfología territorial en la que impera la periurbanización y la policentralidad" (De Mattos, 2004: 19). Éste último relacionado con la expansión territorial de áreas metropolitanas y la aparición de nuevos centros urbanos.

En relación a esta morfología territorial, Ascher (2004) señala que, en un primer momento, el crecimiento urbano de las ciudades tiene manifestaciones internas, las cuales están asociadas a la extensión de las aglomeraciones a su periferia más inmediata y un crecimiento externo vinculado a la integración de ciudades alejadas, y que funcionaban de manera independiente a la zona de funcionamiento metropolitano. Este proceso de urbanización tendría consecuencias directas en las relaciones que se dan en un espacio cotidiano, fomentando la privatización y fragmentación de estos, en donde "lo local cambia de naturaleza y sentido" (De Mattos, 2004: 22).

Un ejemplo de estas transformaciones urbanas se refleja en el Área Metropolitana de Santiago, donde la extensión hacia la periferia implicó ciertos cambios, como en el caso de Puente Alto, San Bernardo y Maipú, los cuales pasan a constituir subcentros de la región, "reforzando sus antiguas funciones centrales con el agregado de nuevas modalidades comerciales" (De Mattos, 2004: 35). 
Con todos estos cambios y transformaciones que sufren las ciudades al alero de los principios neoliberales, surgen diversas interrogantes en relación a las formas en que se piensan y se construyen las ciudades, las implicancias de los modos de gestión a nivel territorial y social, y cómo se entiende el espacio público (las relaciones que se establecen en estos espacios y sus significaciones).

En este sentido, las nuevas regiones metropolitanas cuestionan nuestra idea de ciudad: "son vastos territorios de urbanización discontinua, fragmentada en unos casos, difusa en otros, sin límites precisos, con escasos referentes físicos y simbólicos que marquen el territorio" (Borja, 2010: 32).

En otras palabras, a partir del desarrollo capitalista de la ciudad, se fomenta y promueve la formación de espacios urbanizados, sin embargo cabe la duda respecto a las características y calidad de estos espacios no solo en su forma física, sino que también por su capacidad de crear ciudad y ciudadanía, lo que al parecer ha quedado en un segundo plano considerando los efectos de estas transformaciones urbanas.

En este sentido, el problema que da origen tanto a la intervención como a la investigación se relaciona con la pérdida del valor social que afecta a la ciudad y a los espacios públicos, ante esta constante mercantilización del territorio. De esta forma, es preciso preguntarse ¿cómo influyen los espacios públicos en la construcción de las ciudades y qué percepción tienen los ciudadanos sobre este proceso? Si bien existen variadas discusiones respecto a qué entendemos por espacios públicos es preciso comprender qué es este espacio en sus múltiples funciones y características.

\section{Casos Villa San Luis (Renca) y Villa El Almendral (Puente Alto)}

Ambos casos fueron seleccionados a partir de la intervención realizada por Fundación Mi Parque, donde ésta responde a la necesidad identificada por este organismo, es decir, el déficit de áreas verdes percibido en cada comuna. 
Si nos detenemos en el Área Metropolitana de Santiago y las características de las áreas verdes, es posible observar que estos espacios se caracterizan por una desigual e inequitativa distribución, tanto en términos de superficie total como de tamaño y accesibilidad. Esta "desigualdad está altamente correlacionada con el nivel socioeconómico de la población: al comparar las comunas (...) se encuentra que cuanto mayor es el nivel de ingresos de los hogares, éstos cuentan con mayor superficie total de áreas verdes, las cuales tienen mayor tamaño y mejor accesibilidad" (Reyes \& Figueroa, 2010: 105).

De esta forma, se justifica el accionar de la fundación y la elección de las comunas mencionadas, dejando en evidencia la falta de equipamiento público y áreas verdes en comunas de la periferia del Área Metropolitana de Santiago. Si tomamos en cuenta el Índice de Calidad de Vida Urbana (ICVU) ${ }^{5}$, también es posible observar cómo se reflejan estas diferencias, ya que ambas comunas se encuentran bajo el promedio nacional, clasificándose a Renca con un ICVU insuficiente $(44,81)$ y Puente Alto con un ICVU bajo $(35,00)$. Además, concentran gran cantidad de viviendas sociales, conformando barrios caracterizados por una composición social homogénea, con débil oferta de comercio y servicios, comparados con el promedio de la ciudad, y alejados de los centros urbanos (Plataforma urbana, 2012).

Al mismo tiempo, respecto a los barrios escogidos, también es posible observar ciertos elementos que caracterizan a la Villa San Luis (Renca) y la Villa El Almendral (Puente Alto). La villa San Luis es un conjunto de vivienda social construido el año 2002 y está ubicado a los pies del cerro Renca. La intervención realizada junto a Fundación Mi Parque consistió en la ejecución de una serie de talleres de diseño participativo y posteriormente la recons-

Este mide "Las condiciones de vida objetivas de la población generadas a partir de las actuaciones y dinámicas de transformación del espacio urbano inducidas por actores públicos, privados y la sociedad civil" (Orellana, 2013: 3). 
trucción de la plaza del sector junto a la comunidad. Este proyecto se realiza en enero del año 2011.

La Villa El Almendral se ubica en el sector de Bajos de Mena, consolidándose como la tercera etapa de un conjunto de viviendas entregadas el año 2002. La intervención junto a Mi Parque fue realizada en septiembre del año 2012, ejecutándose talleres de diseño participativo y la reconstrucción de la plaza junto a los vecinos del sector.

\section{Función social del espacio público}

Considerando el proceso de mercantilización de las ciudades surgen diferentes interrogantes respecto a cómo es la relación que se construye con el espacio público y su capacidad para crear ciudad y ciudadanía, es decir, cómo se construye la relación con el territorio y con un otro distinto.

El espacio público se ha visto como un lugar físico de dominio público. Esta concepción hace alusión a una mirada restrictiva de este concepto, destacando la separación entre lo privado y lo público, en donde lo público se convierte en un espacio residual o marginal, como por ejemplo, un espacio que quedó después de la vivienda. En otras palabras, el espacio público es lo opuesto al espacio privado.

Desde un punto de vista jurídico, el espacio público seria aquel que está "sometido a una regulación específica por parte de la administración pública, propietaria o que posee la facultad de dominio del suelo, que garantiza su accesibilidad a todos y fija las condiciones de su utilización y de instalación de actividades" (Segovia y Jordán, 2005: 17).

Por otro lado, también es posible encontrar conceptualizaciones en cuanto a los usos, donde el espacio público tiene la función de relacionar, de crear lugares de esparcimiento, de identificación y centralidad.

Algunas conceptualizaciones destacan la dimensión histórica del espacio público, el cual "no se agota ni está asociado únicamente a 
lo físico-espacial, sea de una unidad o de un sistema de espacios. Es más bien, un ámbito contenedor de la conflictividad social, que tiene distintas posiciones dependiendo de la coyuntura y de la ciudad que se trate" (Carrión, 2002: 3).

Sin embargo, estos espacios estarían en directa relación con la ciudad, por lo que no se pueden entender sin establecer esta conexión histórica entre ambos, ya que las transformaciones están dadas por el contexto en el que se sitúa. Es decir, este espacio no solo es construido estructuralmente ${ }^{6}$, sino que también en términos sociales, dependiendo de la relación que los habitantes establecen con él. Este espacio "abriga la capacidad de los grupos para organizarse, soñar juntos e implementar acciones de bien común; y en su dimensión política, representa un lugar de encuentro de ideas y de transparencia" (Oviedo \& Abogabir, 2000: 19). En función de la dimensión política e histórica a la que se hace alusión, en lo público se puede observar y manifestar más claramente la crisis de la ciudad, reflejando el modelo de planificación urbana actual y los efectos de estos procesos en la calidad de vida de sus habitantes.

A partir de esto, es posible observar cómo el carácter subjetivo o simbólico del espacio, entendiendo por ello la trama de percepciones, memorias o sentimientos que guían nuestro accionar (Güell, 2002), es el que va permeando la construcción de lo público, aquello que a su vez tiene la facultad de definirlo como tal. Al mismo tiempo, este espacio debe contar con ciertas características que faciliten y promuevan el contacto y la identificación entre los sujetos. En este sentido, se refuerza y promueve la idea de que son los usos que le asignan los sujetos a un espacio lo que le otorga el carácter de público (Borja \& Muxi, 2003).

Es necesario tener en cuenta que estos procesos de construcción social del espacio público se pueden desarrollar o no, ya que como se menciona anteriormente, las dinámicas de globalización y

Entendiéndose el elemento estructural como la construcción físico-material del espacio público. 
segregación no contribuyen a generar procesos de identificación con un territorio o con otro. Por lo tanto, la apropiación de los espacios también estaría determinada por la dimensión física del barrio.

En rigor, el espacio público no solo podría configurarse como sinónimo de calidad urbana, sino que también es un elemento indispensable para hacer ciudad; es por ello que sería necesario considerar las periferias, construir nuevas centralidades y otorgar un valor ciudadano a la política urbanística (Borja \& Muxi, 2003). En conclusión, lo público se constituye en la articulación de su dimensión social y física.

\section{Espacio público como elemento estructurador}

En función de lo señalado en apartados anteriores, es posible observar cómo el espacio público no solo se encuentra determinado por una función física, sino que también tiene la capacidad de incidir en las formas de habitar. Esta situación permite situar al espacio público como un elemento estructurador de los barrios. En este sentido, es necesario tener en cuenta la imagen que tienen los sujetos respecto a este espacio para poder comprender y observar la relación entre estructura y configuración social de espacio público, ya que, todo "ciudadano tiene largos vínculos con una u otra parte de su ciudad, y su imagen está embebida de recuerdos y significados" (Lynch, 1998: 9).

Es por esto que para cada sujeto su experiencia en la ciudad puede tener diferentes significados, lo que refleja su carácter móvil. No somos tan solo "observadores de este espectáculo, sino que también somos parte de él, y compartimos el escenario con los demás participantes" (Lynch, 1998: 10). Por lo tanto, se entiende que la ciudad no es concebida como un objeto, sino que se configura a través de las percepciones que los sujetos tienen de ella.

Al mismo tiempo, se refleja no solo la función física de la ciudad, ya que, un "escenario físico vívido e integrado, capaz de generar una imagen nítida, desempeña así mismo una función social. 
Puede proporcionar la materia prima para los símbolos y recuerdos colectivos de comunicación de un grupo" (Lynch, 1998: 13). Esta imagen, a su vez, tiene la capacidad de definir y configurar un espacio como seguro, y no solo otorga seguridad al espacio, sino que también aporta profundidad y significados a las relaciones que se establecen entre los sujetos.

De esta forma, es posible distinguir ciertos elementos que contribuyen a estructurar la imagen de la ciudad o del barrio que habita el sujeto. Estos corresponden a sendas, barrios, nodos (núcleos) y bordes. En rigor, a través de esta dimensión física es preciso observar si los elementos que están en su base se comportan como espacio público; en otras palabras, cuál es la función que desempeñan. Es preciso tener en cuenta que estos elementos no pueden ser observados de forma particular, sino que se deben leer en su conjunto, ya que éstos forman parte de un todo que responde a la imagen de la ciudad que perciben los sujetos.

\section{¿Cómo es percibido el espacio público?}

A partir de las entrevistas en profundidad y mapas que realizaron los habitantes de la Villa San Luis de Renca y la Villa El Almendral se realizó un proceso de reconstrucción de las categorías de Sendas, Bordes, Nodos y Barrios, con el fin de relevar la relación que tienen los sujetos con su entorno, es decir, la importancia de lo público como elemento estructurador y los significados que adquiere este espacio. La reconstrucción de estas categorías es el diálogo que se produce entre la dimensión física de lo público y su dimensión social. 


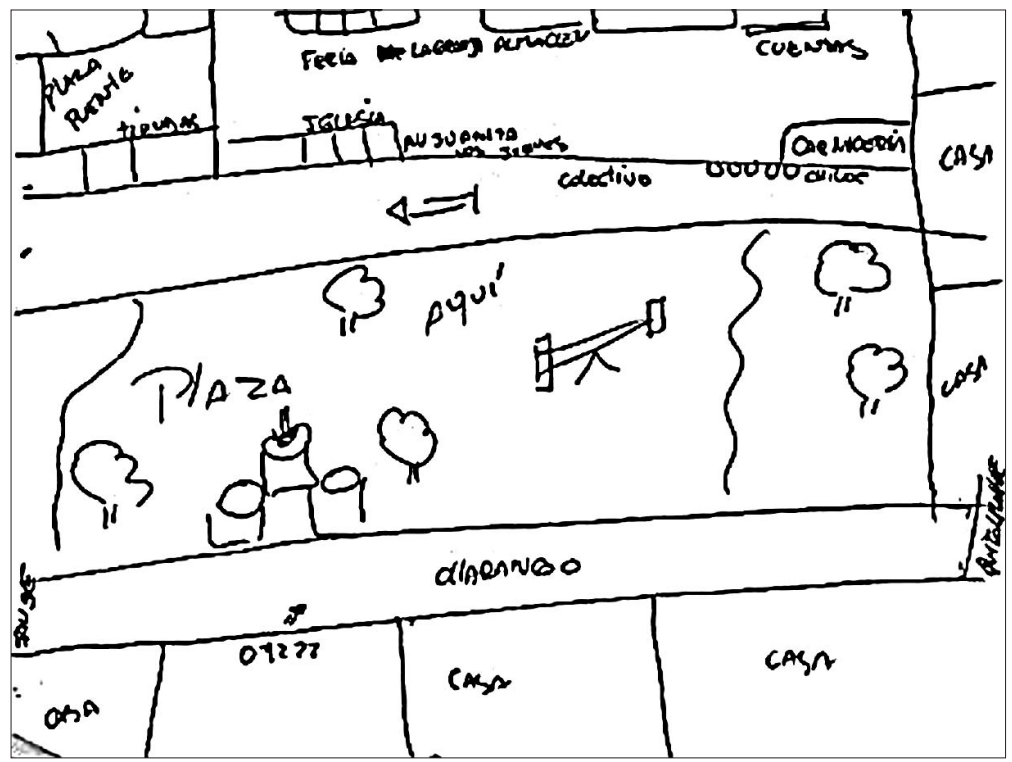

Imagen 1: Mapa Villa El Almendral, Puente Alto. Fuente: Entrevista N³.

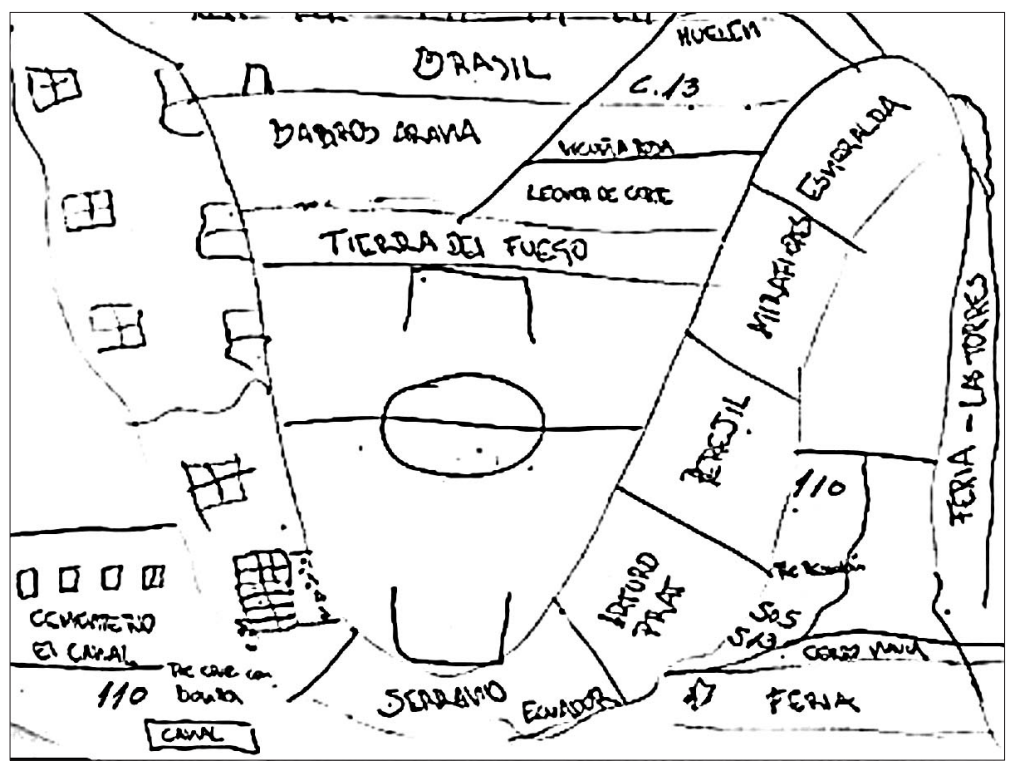

Imagen 2: Mapa Villa San Luis, Renca. Fuente: Entrevista $\mathrm{N}^{\circ} 1$.

Si bien, al parecer las sendas no se presentan como un factor importante si se piensa en su unidad, al comprender esta categoría 
en función de los otros elementos, cobra importancia como parte fundamental de la imagen y estructura del barrio. Es decir, las sendas representan los límites o bordes entre un sector y otro, tanto al interior de la villa como en su exterior.

En este sentido, ocurren situaciones particulares en cada barrio analizado, ya que, por un lado, los límites en la Villa San Luis se presentan al interior de este espacio en relación a los vecinos y a los lugares por donde se puede o no transitar: "si tu pasái pa allá [haciendo referencia a un block de la villa] tú veí la diferencia entre el lado de allá y el lado de acá" $(\mathrm{R}-2)$. Por otro lado, los límites en la Villa El Almendral guardan relación con elementos externos a este espacio, es decir, con los alrededores de la villa: "Ahora aquí casi todo es malo, la villa no... uno aquí puede salir en la noche, sino que alrededor no" (PA - 4). Así, los bordes constituyen un elemento que demarca los espacios por los cuales las personas pueden transitar o no, o que consideran seguros o peligrosos.

En cuanto a los nodos o elementos centrales se percibe una tendencia en torno al repliegue hacia el interior de los barrios. Al respecto, es posible indicar el carácter cotidiano que adquiere la ciudad, ya que los mapas están enfocados en la imagen del barrio, la villa y las experiencias en torno a este espacio. La ciudad que experimentan los sujetos, se desarrolla en los espacios de uso frecuente. Este hecho genera distintas interrogantes, considerando el contexto en el que nos situamos. ¿Asistimos a una progresiva privatización de los espacios públicos?

Tomando en cuenta las categorías analizadas, es posible observar que éstas confluyen en un elemento central que es capaz de articular y contener tanto sendas, como bordes y nodos. En este sentido, los barrios adquieren un rol principal en la estructura e imagen que los sujetos perciben respecto a su ciudad y a los espacios públicos.

Tanto en los mapas como en el discurso de los sujetos, se observa la influencia que tienen las experiencias en torno a los significados del habitar en un determinado barrio, en otras palabras, cómo 
estas experiencias van construyendo las formas de percibir e identificarse con el territorio y con otro distinto.

A pesar de estas diferencias, en cuanto al sentimiento de arraigo o vínculos que pueden establecer con el territorio los sujetos que residen en él, en ambos casos se observa un vínculo débil con sus pares y con el espacio físico. Si bien en la Villa El Almendral la reconstrucción de la plaza del barrio significó que los vecinos se conocieran entre sí y se motivaran a cuidar el espacio físico que los rodea, manifiestan que si tuvieran la posibilidad de estar en otro barrio, lo harían, no exclusivamente por el hecho de habitar esa Villa, sino por las dinámicas que se dan en el exterior de este espacio, también por el deseo de estar más cerca del centro urbano "me gusta el barrio, pero igual me cambiaría más cerca del centro de Puente Alto" (PA - 2).

Por otro lado, en la Villa San Luis no existen vínculos con el territorio, lo cual se puede producir por las experiencias que viven en este espacio: marcado por el miedo y temor hacia ciertos sectores de la villa y las relaciones que se articulan con los sujetos que habitan en él: "Es que uno no tiene la posibilidad de andar caminando tranquilamente, yo si la tuviera, no tengo por qué dejar mi casa, pero no se puede... si erradicaran eso [haciendo alusión a un block de la villa] esto tendría más valor" ( $\mathrm{R}-3)$. Lo anterior no permitiría construir un espacio de identificación simbólica con el territorio, el espacio público reconstruido y la comunidad.

\section{Aportes para la sustentabilidad social}

Es preciso considerar que la sustentabilidad de los espacios públicos no solo se relaciona con su dimensión física-estructural o mantención de estos espacios, sino que se entiende como la relación que establecen los sujetos con el territorio, reconociendo la capacidad de generar espacios de autonomía y autorregulación comunitaria en donde las inversiones urbanas respondan a las demandas sociales identificadas (Acselrad, 1999). En suma, la sustentabilidad depende y se define por los sujetos. 
En relación a la imagen que construyen los sujetos respecto al territorio que habitan y los significados que adquieren estos espacios, se observa cómo el relato de los entrevistados va dando forma y sentido a los conceptos que están en la base de la intervención realizada, donde el objetivo apunta a la comprensión del discurso de los vecinos que habitan el barrio respecto al espacio público reconstruido. Es decir, permite una comprensión compleja sobre los espacios públicos y las relaciones que se desarrollan en éstos, y la relevancia que tiene para los sujetos en la imagen que perciben de su ciudad. En otras palabras, se abre la discusión en relación a cómo conciben el espacio público reconstruido y si este elemento se constituye como un factor estratégico y central en su imagen o más bien representa un borde o límite respecto al lugar en el que residen.

Esta aproximación al discurso de las personas que habitan cada Villa, se observa como una necesidad para integrar sus percepciones respecto al hito que marca la reconstrucción de la plaza de su barrio y si este hecho guarda relación con la imagen que tienen de los espacios públicos que frecuentan a nivel macro. Esto marca una de las principales articulaciones entre el proyecto de intervención e investigación, que se da a nivel discursivo y conceptual, integrando las percepciones en torno al espacio reconstruido y el espacio público en una escala global.

En rigor, es posible mencionar que no solamente estas percepciones se relacionan o se ven permeadas por este contexto de mercantilización y segregación de las ciudades, sino que también guardan relación con las dimensiones físicas y estructurales del territorio en particular. En otras palabras, estas memorias o sentimientos compartidos se configuran dentro del espacio local como por ejemplo "la villa", "manzana", "mi calle", por lo que depende de ese contexto particular la relación que se establece con el espacio público.

Entonces, el espacio público puede concebirse como un lugar de resguardo en relación con lo que ocurre en el entorno o como un 
espacio del que "hay que cuidarse", por lo que este contexto es el que va permeando la construcción de las subjetividades de un barrio, o si permite o no dicho proceso. Sin embargo, esto no quiere decir que estos barrios no se sitúen en un contexto de segregación, sino que se da una relación dialéctica entre lo local y lo global.

Un segundo elemento tiene que ver con las personas, las comunidades y las aproximaciones que realizan según su posición en el territorio, por ejemplo, si bien se desarrolla la idea de que los sujetos pueden construir diferentes formas de aproximación a los espacios públicos y a su ciudad, se refuerza la imagen de que quienes definen un espacio como público son quienes le otorgan ciertos usos; en este sentido, y en directa relación con el proceso de intervención, quienes tienen el poder de determinar las características de apropiación positiva o negativa son ellos y no puede ser clasificado a priori.

\section{Conclusiones}

Las articulaciones del proceso de intervención e investigación permiten abrir distintas reflexiones en torno a lo público y los significados que adquiere dependiendo de los sujetos que lo habitan. Si bien se observa el carácter local de la ciudad y de los espacios públicos, pareciera cobrar importancia que lo público no responde sólo a un cambio físico del territorio o al carácter funcional de éste, sino que la configuración de estos espacios debe responder a un cambio de perspectiva, como un espacio donde uno se reconoce y otorga distintos significados, lo cual invita a hacerse responsable de él.

A su vez el proceso de intervención e investigación permitió problematizar conceptos que se encuentran en la base de la intervención que realiza Fundación Mi Parque, como lo es la sustentabilidad social. Estas interrogantes se vinculan con la idea de profundizar respecto a quiénes son los encargados de la sustentabilidad social de lo público y cómo promover la relevancia de lo social como una solución de los aspectos físicos del territorio. Si bien 
la sustentabilidad social estaría dada por los sujetos en la medida que el espacio público responda a las necesidades e intereses de la comunidad, es preciso que las intervenciones en torno al espacio público se cuestionen el para qué y para quiénes están orientadas, invirtiendo la primacía de los aspectos físicos por los símbolos y significaciones de dicha intervención. El presente proyecto releva la importancia de la relación entre conocimiento y acción, donde las percepciones de la comunidad permiten dotar de significado a las acciones relacionadas con el mejoramiento de los espacios públicos.

Este cuestionamiento por los significados de lo público invita a realizar un ejercicio crítico respecto a la desigualdad o inequidad social que puede reflejar este espacio. Si bien la distribución y las características de lo público tendrían relación con el perfil socioeconómico de los sujetos que habitan este espacio, se observa la relevancia o el impacto que tiene lo público como la base del cuestionamiento por la justicia social respecto al derecho que tiene la ciudadanía por residir en "un lugar bonito". Es decir, los significados que adquiere el espacio público permiten abrir la discusión en torno a cómo construimos nuestras ciudades y quiénes participan en dicha construcción, discusión que parte en un ámbito local donde se reconocen construcciones y significaciones comunes.

Si bien se refleja la importancia de la dimensión local de lo público como espacio próximo que permite la identificación con el territorio y la definición del lugar que ocupamos en la ciudad, no se puede olvidar la facultad que tiene este espacio para conectar diferentes territorios (Borja y Muxí, 2003). Es decir, lo público se construye en la relación entre lo local y lo global, y cómo a partir de la intervención en un espacio cotidiano se puede fomentar la apropiación y el vínculo que construimos con la ciudad que se habita. En conclusión, existe un doble desafío, la reconstrucción del espacio público local en función de las significaciones de los sujetos para con el territorio y cómo este espacio es capaz de articular lo público en términos globales. 
Espacio público: en busca de nuevos significados y estrategias de sustentabilidad social. Un estudio comparado de dos barrios / Valderrama

\section{Bibliografía}

Acselrad, H. (1999). Sustentabilidad y ciudad. Revista EURE, 25 (74), 35-46.

Ascher, F. (2004). Los nuevos principios del urbanismo. Madrid: Alianza Editorial.

Borja, J. (2010). La democracia en busca de la ciudad futura. En Sugranyes, A. y Mathivet, C. (Edits.), Ciudades para tod@s: por el derecho a la ciudad, propuestas y experiencias (pp. 29-45). Santiago.

Borja, J., \& Muxi, Z. (2003). El espacio público: ciudad y ciudadanía. Barcelona: Electa.

Carrión, F. (2002). Espacio público: punto de partida para la alteridad, en Segovia (edit.), Espacios públicos y construcción social. Hacia un ejercicio de ciudadanía. Santiago, Ediciones Sur.

Güell, P. (2002). Subjetividad social: desafío para el nuevo siglo. Polis.

Lynch, K. (1998). La imagen de la ciudad. Barcelona: Editorial Gustavo Gili.

Mattos, C. (2004). Santiago de Chile: Metamorfosis bajo un nuevo impulso de modernización capitalista. En Mattos, C., Ducci, M., Rodríguez, A. y Yáñez, G. (Edits.), Santiago en la Globalización: ¿una nueva ciudad? (pp. 17-44). Santiago: SUR.

Orellana (2013) Índice de calidad de vida urbana.

Oviedo, E. \& Abogabir, X. (2000). Participación Ciudadana y Espacio Público. En Segovia, O. y Dascal, G. (Edits.) Espacio público, participación y ciudadanía (pp. 19-34). Santiago: Sur.

Plataforma Urbana (2012). Estudio de Vivienda: 25 comunas concentran departamentos tipo block como vivienda. Disponible en: socialhttp://www.plataformaurbana.cl/archive/2012/05/10/ estudio-de-vivienda-25-comunas-concentrandepartamentos-tipo-block-como-vivienda-social/

Reyes, S. \& Figueroa, I. (2010). Distribución, superficie y accesibilidad de las áreas verdes en Santiago de Chile. Revista EURE, 36(109), 89-110.

Rodríguez, A. y Rodríguez, P. (2012). Políticas neoliberales en Santiago de Chile: políticas contra la ciudad. En Belil, M., Borja, J. y Corti, M. (Edits.) Ciudades, una ecuación imposible (pp. 131-153). Buenos Aires: Icaria.

Segovia, O. y Jordán, R. (2005). Espacios públicos urbanos, pobreza y construcción social. CEPAL. 